\title{
Novel Deleterious nsSNPs within MEFV Gene that Could Be Used as Diagnostic Markers to Predict Hereditary Familial Mediterranean Fever: Using Bioinformatics Analysis
}

\author{
Mujahed I. Mustafa $\mathbb{D}^{1,2}$ Tebyan A. Abdelhameed $\mathbb{D}^{1},{ }^{2}$ Fatima A. Abdelrhman, ${ }^{2}$ \\ Soada A. Osman, ${ }^{2}$ and Mohamed A. Hassan ${ }^{2}$ \\ ${ }^{1}$ Department of Biochemistry, University of Bahri, Sudan \\ ${ }^{2}$ Department of Biotechnology, Africa City of Technology, Sudan
}

Correspondence should be addressed to Mujahed I. Mustafa; mujahedibrahim44@gmail.com

Received 29 September 2018; Revised 2 January 2019; Accepted 21 January 2019; Published 4 June 2019

Academic Editor: Nurit Haspel

Copyright ( 2019 Mujahed I. Mustafa et al. This is an open access article distributed under the Creative Commons Attribution License, which permits unrestricted use, distribution, and reproduction in any medium, provided the original work is properly cited.

\begin{abstract}
Background. Familial Mediterranean Fever (FMF) is the most common autoinflammatory disease (AID) affecting mainly the ethnic groups originating from Mediterranean basin. We aimed to identify the pathogenic SNPs in MEFV by computational analysis software. Methods. We carried out in silico prediction of structural effect of each SNP using different bioinformatics tools to predict substitution influence on protein structure and function. Result. 23 novel mutations out of $857 \mathrm{nsSNPs}$ are found to have deleterious effect on the MEFV structure and function. Conclusion. This is the first in silico analysis of MEFV gene to prioritize SNPs for further genetic mapping studies. After using multiple bioinformatics tools to compare and rely on the results predicted, we found 23 novel mutations that may cause FMF disease and it could be used as diagnostic markers for Mediterranean basin populations.
\end{abstract}

\section{Introduction}

Familial Mediterranean Fever is an autosomal recessive inherited inflammatory disease [1-3] (however, it has been observed that a substantial number of patients with clinical FMF possess only one demonstrable MEFV mutation [4, 5]) that is principally seen in different countries [6-10]. However, patients from different ethnicities (such as Japan) are being increasingly recognized $[2,11]$, and the carrier frequency for $M E F V$ genetic variants in the population in the Mediterranean basin is about $8 \%$ [12]. Most cases of FMF usually present with acute abdominal pain and fever $[1,3,7]$, both of which are also the main causes of referral in the emergency department [13]. All these factors may help in medical treatment. Colchicine is the first line therapy [14], but in resistant cases $(<10 \%$ of patients) [15], it affects the responsiveness to Colchicine [16]; other anti-inflammatory drugs can be used for extra anti-inflammatory effect [17]. If FMF is not treated, it may be an etiologic factor for colonic LNH in children [18]. MEFV gene is localized on
16 p13.3 of chromosome 16 at position 13.3 which consists of 10 exons with $21600 \mathrm{bp}[3,19]$. The disease is characterized by recurrent febrile episodes and inflammation in the form of sterile polyserositis. Amyloid protein involved in inflammatory amyloidosis was named AA (amyloid-associated) protein and its circulating precursor was named SAA (serum amyloid-associated). Amyloidosis of the AA type is the most severe complication of the disease. The gene responsible for FMF, $M E F V$, encodes a protein called pyrin or marenostrin and is expressed mainly in neutrophils $[3,19]$.

The definition of the $M E F V$ gene has permitted genetic diagnosis of the disease. Nevertheless, as studies have unwrapped molecular data, problems have arisen with the clinical definitions of the disease [20]. FMF is caused by mutations in the MEFV missense SNPs (we were focusing on SNPs which are located in the coding region because it is much important in disease causing potential, which are responsible for amino acid residue substitutions resulting in functional diversity of proteins in humans) [20] coding for pyrin, which is a component of inflammasome functioning 
in inflammatory response and production of interleukin- $1 \beta$ (IL-1 $\beta)$. Recent studies have shown that pyrin recognizes bacterial modifications in Rho GTPases, which results in inflammasome activation and increase in IL-1 $\beta$. Pyrin does not directly recognize Rho modification but probably is affected by Rho effector kinase, which is a downstream event in the actin cytoskeleton pathway [19, 21, 22].

The aim of this study was to identify the pathogenic SNPs in $M E F V$ using in silico prediction software and to determine the structure, function, and regulation of their respective proteins. This is the first in silico analysis in $M E F V$ gene to prioritize SNPs for further genetic mapping studies. The usage of in silico approach has strong impact on the identification of candidate SNPs since they are easy and less costly and can facilitate future genetic studies [23].

\section{Method}

2.1. Data Mining. The data on human $M E F V$ gene was collected from National Center for Biological Information (NCBI) website [24]. The SNP information (protein accession number and SNP ID) of the $M E F V$ gene was retrieved from the NCBI dbSNP (http://www.ncbi.nlm.nih.gov/snp/) and the protein sequence was collected from Swiss Prot databases (http://expasy.org/) [25].

2.2. SIFT. SIFT is a sequence homology-based tool [26] that sorts intolerant from tolerant amino acid substitutions and predicts whether an amino acid substitution in a protein will have a phenotypic effect. It considers the position at which the change occurred and the type of amino acid change. Given a protein sequence, SIFT chooses related proteins and obtains an alignment of these proteins with the query. Based on the amino acids appearing at each position in the alignment, SIFT calculates the probability that an amino acid at a position is tolerated conditional on the most frequent amino acid being tolerated. If this normalized value is less than a cutoff, the substitution is predicted to be deleterious. SIFT scores $<0.05$ are predicted by the algorithm to be intolerant or deleterious amino acid substitutions, whereas scores $>0.05$ are considered tolerant. It is available at (http://sift.bii.astar.edu.sg/).

2.3. PolyPhen-2. It is a software tool [27] to predict possible impact of an amino acid substitution on both structure and function of a human protein by analysis of multiple sequence alignment and protein 3D structure; in addition, it calculates position-specific independent count scores (PSIC) for each of the two variants and then calculates the PSIC scores difference between the two variants. The higher a PSIC score difference is, the higher the functional impact a particular amino acid substitution is likely to have. Prediction outcomes could be classified as probably damaging, possibly damaging or benign according to the value of PSIC as it ranges from (0_1); values closer to zero were considered benign while values closer to 1 were considered probably damaging and also it can be indicated by a vertical black marker inside a color gradient bar, where green is benign and red is damaging. nsSNPs that is predicted to be intolerant by SIFT has been submitted to PolyPhen as protein sequence in FASTA format obtained from UniproktB/Expasy after submitting the relevant ensemble protein (ESNP) there, and then we entered position of mutation, native amino acid, and the new substituent for both structural and functional predictions. PolyPhen version 2.2.2 is available at http://genetics.bwh.harvard.edu/pph2/index.shtml.

2.4. Provean. Provean is a software tool [28] which predicts whether an amino acid substitution or indel has an impact on the biological function of a protein. It is useful for filtering sequence variants to identify nonsynonymous or indel variants that are predicted to be functionally important. It is available at (https://rostlab.org/services/snap2web/).

2.5. SNAP2. Functional effects of mutations are predicted with SNAP2 [29]. SNAP2 is a trained classifier that is based on a machine learning device called "neural network". It distinguishes between effect and neutral variants/nonsynonymous SNPs by taking a variety of sequence and variant features into account. The most important input signal for the prediction is the evolutionary information taken from an automatically generated multiple sequence alignment. Also structural features such as predicted secondary structure and solvent accessibility are considered. If available also annotation (i.e., known functional residues, pattern, regions) of the sequence or close homologs are pulled in. In a cross-validation over 100,000 experimentally annotated variants, SNAP2 reached sustained two-state accuracy (effect/neutral) of $82 \%$ (at an AUC of 0.9). In our hands this constitutes an important and significant improvement over other methods. It is available at (https://rostlab.org/services/snap2web/).

2.6. PHD-SNP. An online Support Vector Machine (SVM) based classifier is optimized to predict if a given single point protein mutation can be classified as disease related or as a neutral polymorphism. It is available at (http://snps.biofold.org/phd-snp/phd-snp.html).

2.7. SNP\&Go. SNPs\&GO is an algorithm developed in the Laboratory of Biocomputing at the University of Bologna directed by Prof. Rita Casadio. SNPs\&GO is an accurate method that, starting from a protein sequence, can predict whether a variation is disease related or not by exploiting the corresponding protein functional annotation. SNPs\&GO collects in unique framework information derived from protein sequence, evolutionary information, and function as encoded in the Gene Ontology terms and outperforms other available predictive methods [30]. It is available at (http://snps.biofold.org/snps-and-go/snps-and-go.html).

2.8. P-Mut. P-MuT, a web-based tool [31] for the annotation of pathological variants on proteins, allows the fast and accurate prediction (approximately $80 \%$ success rate in humans) of the pathological character of single point amino acidic mutations based on the use of neural networks. It is available at (http://mmb.irbbarcelona.org/PMut). 
TABLE 1: Damaging or deleterious effect nsSNPs associated variations predicted by various softwares.

\begin{tabular}{|c|c|c|c|c|c|c|c|c|}
\hline \multirow{2}{*}{ Amino Acid Change } & \multicolumn{2}{|l|}{ SIFT } & \multicolumn{2}{|l|}{ Polyphen } & \multicolumn{2}{|r|}{ PROVEAN } & \multicolumn{2}{|c|}{ SNAP2 } \\
\hline & prediction & Score & Prediction & score & Score & Prediction $($ cutoff $=-2.5)$ & prediction & score \\
\hline S749Y & DAMAGING & 0 & PROBABLY DAMAGING & 1.000 & -3.116 & Deleterious & effect & 64 \\
\hline F743S & DAMAGING & 0 & PROBABLY DAMAGING & 1.000 & -5.563 & Deleterious & effect & 68 \\
\hline Y741C & DAMAGING & 0 & PROBABLY DAMAGING & 1.000 & -6.035 & Deleterious & effect & 77 \\
\hline F731V & DAMAGING & 0 & PROBABLY DAMAGING & 1.000 & -5.159 & Deleterious & effect & 81 \\
\hline I720T & DAMAGING & 0 & PROBABLY DAMAGING & 1.000 & -3.639 & Deleterious & effect & 58 \\
\hline L709R & DAMAGING & 0 & PROBABLY DAMAGING & 1.000 & -4.311 & Deleterious & effect & 77 \\
\hline V691G & DAMAGING & 0 & PROBABLY DAMAGING & 1.000 & -4.667 & Deleterious & effect & 66 \\
\hline W689R & DAMAGING & 0 & PROBABLY DAMAGING & 1.000 & -10.132 & Deleterious & effect & 89 \\
\hline G668R & DAMAGING & 0 & PROBABLY DAMAGING & 1.000 & -6.287 & Deleterious & effect & 92 \\
\hline V659F & DAMAGING & 0 & PROBABLY DAMAGING & 1.000 & -3.811 & Deleterious & effect & 64 \\
\hline F636C & DAMAGING & 0 & PROBABLY DAMAGING & 1.000 & -6.49 & Deleterious & effect & 79 \\
\hline R461W & DAMAGING & 0 & PROBABLY DAMAGING & 1.000 & -5.456 & Deleterious & effect & 68 \\
\hline H407Q & DAMAGING & 0 & PROBABLY DAMAGING & 1.000 & -7.335 & Deleterious & effect & 41 \\
\hline H407R & DAMAGING & 0 & PROBABLY DAMAGING & 1.000 & -7.332 & Deleterious & effect & 51 \\
\hline $\mathrm{H} 404 \mathrm{R}$ & DAMAGING & 0 & PROBABLY DAMAGING & 1.000 & -7.349 & Deleterious & effect & 75 \\
\hline C398Y & DAMAGING & 0 & PROBABLY DAMAGING & 1.000 & -10.314 & Deleterious & effect & 51 \\
\hline C395Y & DAMAGING & 0 & PROBABLY DAMAGING & 1.000 & -10.262 & Deleterious & effect & 19 \\
\hline C395F & DAMAGING & 0 & PROBABLY DAMAGING & 1.000 & -10.315 & Deleterious & effect & 27 \\
\hline C395R & DAMAGING & 0 & PROBABLY DAMAGING & 1.000 & -11.074 & Deleterious & effect & 27 \\
\hline H378Q & DAMAGING & 0 & PROBABLY DAMAGING & 1.000 & -5.886 & Deleterious & effect & 38 \\
\hline H378Y & DAMAGING & 0 & PROBABLY DAMAGING & 1.000 & -4.884 & Deleterious & effect & 45 \\
\hline C375R & DAMAGING & 0 & PROBABLY DAMAGING & 1.000 & -8.429 & Deleterious & effect & 66 \\
\hline L86P & DAMAGING & 0 & PROBABLY DAMAGING & 1.000 & -4.1 & Deleterious & effect & 19 \\
\hline
\end{tabular}

2.9. I-Mutant 3.0. I-Mutant 3.0 is a neural network based tool [32] for the routine analysis of protein stability and alterations by taking into account the single-site mutations. The FASTA sequence of protein retrieved from UniProt is used as an input to predict the mutational effect on protein stability. It is available at (http://gpcr2.biocomp.unibo.it/ cgi/predictors/I-Mutant3.0/I-Mutant3.0.cgi).

2.10. Modeling nsSNP Locations on Protein Structure. Project hope is a new online web-server to search protein $3 \mathrm{D}$ structures (if available) by collecting structural information from a series of sources, including calculations on the 3D coordinates of the protein, sequence annotations from the UniProt database, and predictions by DAS services. Protein sequences were submitted to project hope server in order to analyze the structural and conformational variations that have resulted from single amino acid substitution corresponding to single nucleotide substitution. It is available at (http://www.cmbi.ru.nl/hope).

2.11. GeneMANIA. We submitted genes and selected from a list of data sets that they wish to query. GeneMANIA's [33] approach is to know protein function prediction integrating multiple genomics and proteomics data sources to make inferences about the function of unknown proteins. It is available at (http://www.genemania.org/).

\section{Results and Discussion}

3.1. Result. See Tables 1-5 and Figure 1.

\section{Discussion}

23 novel mutations have been found (see Table 3) which affected the stability and function of the $M E F V$ gene using bioinformatics tools. The methods used were based on different aspects and parameters describing the pathogenicity and provided clues on the molecular level about the effect of mutations. It was not easy to predict the pathogenic effect of SNPs using single method. Therefore, multiple methods were used to compare and rely on the results predicted. In this study we used different in silico prediction algorithms: SIFT, PolyPhen-2, Provean, SNAP2, SNP\&GO, PHD-SNP, P-MuT, and I-Mutant 3.0 (see Figure 1).

This study identified the total number of nsSNP in Homo sapiens located in coding region of $M E F V$ gene, which were investigated in dbSNP/NCBI Database [24]. Out of 2369, there are 856 nsSNPs (missense mutations) submitted to SIFT server, PolyPhen-2 server, Provean sever, and SNAP2, respectively, and 392 SNPs were predicted to be deleterious in SIFT server. In PolyPhen-2 server, the result showed that 453 were found to be damaging (147 possibly damaging and 306 probably damaging showing deleterious). In Provean server our result showed that 244 SNPs were predicted to be 
TABLE 2: Disease effect nsSNPs associated variations predicted by various softwares.

\begin{tabular}{|c|c|c|c|c|c|c|c|c|}
\hline \multirow{2}{*}{ Amino Acid Change } & \multicolumn{3}{|c|}{ SNP\&GO } & \multicolumn{3}{|c|}{ PHD-SNP } & \multicolumn{2}{|c|}{ P-Mut } \\
\hline & Prediction & RI & Probability & Prediction & RI & Score & Score & Prediction \\
\hline S749Y & Disease & 1 & 0.573 & Disease & 3 & 0.649 & $0.67(85 \%)$ & Disease \\
\hline F743S & Disease & 2 & 0.617 & Disease & 4 & 0.696 & $0.82(90 \%)$ & Disease \\
\hline Y741C & Disease & 6 & 0.797 & Disease & 7 & 0.869 & $0.61(83 \%)$ & Disease \\
\hline F731V & Disease & 6 & 0.79 & Disease & 8 & 0.899 & $0.93(94 \%)$ & Disease \\
\hline $\mathrm{I} 720 \mathrm{~T}$ & Disease & 6 & 0.811 & Disease & 5 & 0.769 & $0.81(89 \%)$ & Disease \\
\hline L709R & Disease & 3 & 0.672 & Disease & 4 & 0.695 & $0.66(85 \%)$ & Disease \\
\hline V691G & Disease & 1 & 0.55 & Disease & 3 & 0.675 & $0.92(93 \%)$ & Disease \\
\hline W689R & Disease & 7 & 0.841 & Disease & 8 & 0.924 & $0.93(94 \%)$ & Disease \\
\hline G668R & Disease & 6 & 0.778 & Disease & 7 & 0.84 & $0.93(94 \%)$ & Disease \\
\hline V659F & Disease & 6 & 0.805 & Disease & 7 & 0.84 & $0.82(90 \%)$ & Disease \\
\hline F636C & Disease & 6 & 0.809 & Disease & 7 & 0.86 & $0.60(82 \%)$ & Disease \\
\hline R461W & Disease & 3 & 0.644 & Disease & 1 & 0.572 & $0.63(84 \%)$ & Disease \\
\hline H407Q & Disease & 6 & 0.788 & Disease & 4 & 0.705 & $0.79(89 \%)$ & Disease \\
\hline H407R & Disease & 5 & 0.769 & Disease & 3 & 0.673 & $0.86(91 \%)$ & Disease \\
\hline H404R & Disease & 5 & 0.744 & Disease & 5 & 0.734 & $0.80(89 \%)$ & Disease \\
\hline C398Y & Disease & 7 & 0.864 & Disease & 8 & 0.912 & $0.86(91 \%)$ & Disease \\
\hline C395Y & Disease & 7 & 0.864 & Disease & 8 & 0.912 & $0.91(93 \%)$ & Disease \\
\hline C395F & Disease & 7 & 0.859 & Disease & 8 & 0.914 & $0.92(94 \%)$ & Disease \\
\hline C395R & Disease & 7 & 0.842 & Disease & 8 & 0.892 & $0.92(94 \%)$ & Disease \\
\hline H378Q & Disease & 4 & 0.714 & Disease & 4 & 0.698 & $0.88(92 \%)$ & Disease \\
\hline H378Y & Disease & 5 & 0.732 & Disease & 5 & 0.728 & $0.80(89 \%)$ & Disease \\
\hline C375R & Disease & 6 & 0.784 & Disease & 6 & 0.822 & $0.92(94 \%)$ & Disease \\
\hline L86P & Disease & 5 & 0.729 & Disease & 6 & 0.801 & $0.51(79 \%)$ & Disease \\
\hline
\end{tabular}

TABLE 3: Stability analysis predicted by I-Mutant version 3.0 (also show the 23 novel mutations).

\begin{tabular}{|c|c|c|c|}
\hline Amino Acid Change & SVM2 Prediction Effect & RI & DDG Value Prediction \\
\hline S749Y & Decrease & 0 & -0.2 \\
\hline F743S & Decrease & 6 & -1.16 \\
\hline Y741C & Decrease & 8 & -2.5 \\
\hline F731V & Decrease & 6 & -1.52 \\
\hline $\mathrm{I} 720 \mathrm{~T}$ & Decrease & 4 & -0.92 \\
\hline L709R & Decrease & 3 & -0.56 \\
\hline V691G & Decrease & 7 & -1.25 \\
\hline W689R & Decrease & 7 & -0.73 \\
\hline G668R & Decrease & 4 & -0.37 \\
\hline V659F & Decrease & 2 & -0.19 \\
\hline F636C & Decrease & 8 & -1.28 \\
\hline $\mathrm{R} 461 \mathrm{~W}$ & Increase & 1 & -0.01 \\
\hline H407Q & Decrease & 8 & -1.48 \\
\hline H407R & Decrease & 5 & -1.1 \\
\hline H404R & Decrease & 1 & -0.06 \\
\hline C398Y & Decrease & 2 & -0.09 \\
\hline C395Y & Increase & 4 & 0.26 \\
\hline C395F & Increase & 1 & 0.04 \\
\hline C395R & Increase & 4 & 0.13 \\
\hline H378Q & Decrease & 4 & -0.68 \\
\hline H378Y & Decrease & 3 & -0.26 \\
\hline C375R & Increase & 2 & -0.01 \\
\hline L86P & Decrease & 2 & -0.56 \\
\hline
\end{tabular}


TABLE 4: The $M E F V$ gene functions and its appearance in network and genome.

\begin{tabular}{|c|c|c|c|}
\hline Function & FDR & Genes in network & Genes in genome \\
\hline nucleotide-binding domain, leucine rich repeat containing receptor signaling pathway & $1.42 \mathrm{E}-07$ & 6 & 47 \\
\hline regulation of interleukin-1 beta production & 0.000129 & 4 & 26 \\
\hline interleukin-1 beta production & 0.000129 & 4 & 30 \\
\hline regulation of interleukin-1 production & 0.000129 & 4 & 30 \\
\hline interleukin-1 production & 0.000196 & 4 & 35 \\
\hline intracellular receptor signaling pathway & 0.000201 & 6 & 207 \\
\hline positive regulation of cysteine-type endopeptidase activity & 0.010438 & 4 & 101 \\
\hline positive regulation of endopeptidase activity & 0.010663 & 4 & 105 \\
\hline positive regulation of peptidase activity & 0.011 & 4 & 109 \\
\hline inflammatory response & 0.018246 & 5 & 283 \\
\hline regulation of chemokine production & 0.018246 & 3 & 39 \\
\hline chemokine production & 0.022338 & 3 & 44 \\
\hline regulation of cysteine-type endopeptidase activity & 0.033238 & 4 & 160 \\
\hline tumor necrosis factor production & 0.033238 & 3 & 54 \\
\hline regulation of tumor necrosis factor production & 0.033238 & 3 & 54 \\
\hline tumor necrosis factor superfamily cytokine production & 0.0407 & 3 & 59 \\
\hline regulation of I-kappaB kinase/NF-kappaB signaling & 0.046902 & 4 & 185 \\
\hline I-kappaB kinase/NF-kappaB signaling & 0.057722 & 4 & 198 \\
\hline positive regulation of cytokine production & 0.065004 & 4 & 207 \\
\hline positive regulation of cysteine-type endopeptidase activity involved in apoptotic process & 0.099763 & 3 & 93 \\
\hline positive regulation of interleukin-1 beta secretion & 0.099763 & 2 & 15 \\
\hline defense response to Gram-negative bacterium & 0.099763 & 2 & 16 \\
\hline cysteine-type endopeptidase activator activity involved in apoptotic process & 0.099763 & 2 & 17 \\
\hline regulation of endopeptidase activity & 0.099763 & 4 & 251 \\
\hline glycosaminoglycan binding & 0.099763 & 3 & 88 \\
\hline regulation of extrinsic apoptotic signaling pathway & 0.099763 & 3 & 92 \\
\hline regulation of peptidase activity & 0.099763 & 4 & 258 \\
\hline positive regulation of interleukin-1 secretion & 0.099763 & 2 & 16 \\
\hline regulation of interleukin-1 beta secretion & 0.099763 & 2 & 17 \\
\hline
\end{tabular}

*FDR: false discovery rate is greater than or equal to the probability that this is a false positive.

deleterious. While in SNAP2 server the result showed that 566 SNPs were predicted to have effect. The differences in prediction capabilities refer to the fact that every prediction algorithm uses different sets of sequences and alignments. In Table 2 we submitted four positive results from SIFT, PolyPhen-2, Provean, and SNAP2 (see Table 1) to observe the disease causing one by SNP\&GO, PHD-SNP, and P-Mut servers.

In SNP\&GO, PHD-SNP and P-Mut softwares were used to predict the association of SNPs with disease. According to SNP\&GO, PHD-SNP and P-Mut (70, 91 and 58 SNPs respectively) were found to be disease-related SNPs. We selected the triple disease-related SNPs only in 3 softwares for further analysis by I-Mutant 3.0, Table 3. I-Mutant result revealed that the protein stability decreased which destabilizes the amino acid interaction (S749Y, F743S, Y741C, F731V, I720T, L709R, V691G, W689R, G668R, V659F, F636C, H407Q, H407R, H404R, C398Y, H378Q, H378Y, and L86P). C375R, C395F, C395R, C395Y, and R461W were found to increase the protein stability (see Table 3 ).
BioEdit software was used to align 10 amino acid sequences of MEFV demonstrating that the residues predicted to be mutated in our band (indicated by red arrow) are evolutionarily conserved across species (see Figure 2). While Project HOPE software was used to submit the 23 most deleterious and damaging nsSNPs (see Figures 3-25), L86P: Proline (the mutant residue) is smaller than Leucine (the wild-type residue); this might lead to loss of interactions. The wild-type and mutant amino acids differ in size. The mutation is located within a domain, annotated in UniProt as Pyrin. The mutation introduces an amino acid with different properties, which can disturb this domain and abolish its function. The wild-type residue is located in a region annotated in UniProt to form an $\alpha$-helix. Proline disrupts an $\alpha$-helix when not located at one of the first 3 positions of that helix. In case of the mutation at hand, the helix will be disturbed and this can have severe effects on the structure of the protein.

GeneMANIA revealed that $M E F V$ has many vital functions: chemokine production, inflammatory response, interleukin-1 beta production, interleukin-1 production, 
TABLE 5: The gene coexpression, shared domain, and interaction with $M E F V$ gene network.

\begin{tabular}{|c|c|c|c|}
\hline Gene 1 & Gene 2 & Weight & Network group \\
\hline PF4 & CEBPB & 0.01083 & Co-expression \\
\hline NLRP14 & MEFV & 0.014663 & Co-expression \\
\hline EPX & PADI4 & 0.01094 & Co-expression \\
\hline CASP1 & PYCARD & 0.012291 & Co-expression \\
\hline TINAGL1 & MEFV & 0.021529 & Co-expression \\
\hline ZNF747 & MEFV & 0.032075 & Co-expression \\
\hline ZNF747 & TINAGL1 & 0.01915 & Co-expression \\
\hline EPX & MEFV & 0.019982 & Co-expression \\
\hline $\mathrm{EPX}$ & ZNF747 & 0.01848 & Co-expression \\
\hline MRPL44 & MEFV & 0.02576 & Co-expression \\
\hline RPL27A & MEFV & 0.023047 & Co-expression \\
\hline TCTN2 & MEFV & 0.02049 & Co-expression \\
\hline TCTN2 & ZNF747 & 0.021219 & Co-expression \\
\hline TCTN2 & RPL27A & 0.019574 & Co-expression \\
\hline ZNF528 & RPL27A & 0.021843 & Co-expression \\
\hline ZNF528 & TCTN2 & 0.020287 & Co-expression \\
\hline PF4 & TINAGL1 & 0.018596 & Co-expression \\
\hline PF4 & EPX & 0.016477 & Co-expression \\
\hline CASP1 & PYCARD & 0.005924 & Co-expression \\
\hline NLRP3 & CEBPB & 0.01342 & Co-expression \\
\hline CASP1 & PYCARD & 0.005896 & Co-expression \\
\hline AZU1 & MEFV & 0.01109 & Co-expression \\
\hline MAP1LC3C & NLRP14 & 0.011062 & Co-expression \\
\hline PADI4 & MEFV & 0.003094 & Co-expression \\
\hline AZU1 & MEFV & 0.003152 & Co-expression \\
\hline AZU1 & PADI4 & 0.004853 & Co-expression \\
\hline ZNF747 & MEFV & 0.004908 & Co-expression \\
\hline PADI4 & MEFV & 0.023362 & Co-expression \\
\hline AZU1 & MEFV & 0.012616 & Co-expression \\
\hline AZU1 & PADI4 & 0.014322 & Co-expression \\
\hline NLRP14 & MEFV & 0.01623 & Co-expression \\
\hline EPX & PADI4 & 0.01024 & Co-expression \\
\hline EPX & AZU1 & 0.007038 & Co-expression \\
\hline ZNF528 & MEFV & 0.039375 & Co-expression \\
\hline PF4 & MEFV & 0.017902 & Co-expression \\
\hline PF4 & AZU1 & 0.012247 & Co-expression \\
\hline PF4 & EPX & 0.007715 & Co-expression \\
\hline TINAGL1 & MEFV & 0.027084 & Co-expression \\
\hline MRPL44 & MEFV & 0.011927 & Co-expression \\
\hline TCTN2 & MEFV & 0.014192 & Co-expression \\
\hline TCTN2 & TINAGL1 & 0.014867 & Co-expression \\
\hline TCTN2 & ZNF747 & 0.010889 & Co-expression \\
\hline TCTN2 & MAP1LC3C & 0.006994 & Co-expression \\
\hline TCTN2 & MRPL44 & 0.010528 & Co-expression \\
\hline ZNF528 & TCTN2 & 0.012167 & Co-expression \\
\hline RPL27A & MEFV & 0.016846 & Co-expression \\
\hline TCTN2 & RPL27A & 0.018021 & Co-expression \\
\hline CASP1 & PSTPIP1 & 0.009518 & Co-expression \\
\hline EPX & AZU1 & 0.01909 & Co-localization \\
\hline PADI4 & MEFV & 0.012301 & Co-localization \\
\hline PADI4 & PSTPIP1 & 0.008748 & Co-localization \\
\hline
\end{tabular}


TABle 5: Continued.

\begin{tabular}{|c|c|c|c|}
\hline Gene 1 & Gene 2 & Weight & Network group \\
\hline AZU1 & MEFV & 0.011852 & Co-localization \\
\hline AZU1 & PSTPIP1 & 0.008052 & Co-localization \\
\hline AZU1 & PADI4 & 0.006025 & Co-localization \\
\hline EPX & MEFV & 0.011933 & Co-localization \\
\hline EPX & PSTPIP1 & 0.008374 & Co-localization \\
\hline EPX & PADI4 & 0.006323 & Co-localization \\
\hline EPX & AZU1 & 0.006061 & Co-localization \\
\hline FBXO9 & MEFV & 0.022287 & Co-localization \\
\hline FBXO9 & PADI4 & 0.009957 & Co-localization \\
\hline FBXO9 & AZU1 & 0.009656 & Co-localization \\
\hline FBXO9 & EPX & 0.009948 & Co-localization \\
\hline PF4 & MEFV & 0.012063 & Co-localization \\
\hline PF4 & PSTPIP1 & 0.007583 & Co-localization \\
\hline PF4 & PADI4 & 0.005603 & Co-localization \\
\hline PF4 & AZU1 & 0.005356 & Co-localization \\
\hline PF4 & EPX & 0.005651 & Co-localization \\
\hline PF4 & FBXO9 & 0.009449 & Co-localization \\
\hline CEBPB & MEFV & 0.159581 & Pathway \\
\hline RELA & MEFV & 0.078321 & Pathway \\
\hline PSTPIP1 & MEFV & 0.953023 & Pathway \\
\hline PYCARD & MEFV & 0.037199 & Pathway \\
\hline CASP1 & PYCARD & 0.037199 & Pathway \\
\hline CASP1 & MEFV & 0.469715 & Physical Interactions \\
\hline NLRP3 & PYCARD & 0.570819 & Physical Interactions \\
\hline PYCARD & MEFV & 0.03673 & Physical Interactions \\
\hline PYCARD & PSTPIP1 & 0.028273 & Physical Interactions \\
\hline CASP1 & PYCARD & 0.017772 & Physical Interactions \\
\hline CASP1 & CEBPB & 0.010941 & Physical Interactions \\
\hline RELA & СЕВPB & 0.00247 & Physical Interactions \\
\hline COG5 & MEFV & 0.211887 & Physical Interactions \\
\hline NLRP3 & MEFV & 0.111467 & Physical Interactions \\
\hline MAP1LC3C & MEFV & 0.104412 & Physical Interactions \\
\hline PYCARD & MEFV & 0.292858 & Physical Interactions \\
\hline NLRP3 & PYCARD & 0.189095 & Physical Interactions \\
\hline PSTPIP1 & MEFV & 0.260595 & Physical Interactions \\
\hline PYCARD & MEFV & 0.204673 & Physical Interactions \\
\hline CASP1 & PYCARD & 0.042335 & Physical Interactions \\
\hline RELA & СЕВPB & 0.007591 & Physical Interactions \\
\hline COG5 & MEFV & 0.387501 & Physical Interactions \\
\hline NLRP3 & PYCARD & 0.304828 & Physical Interactions \\
\hline NLRP3 & PYCARD & 1 & Predicted \\
\hline PYCARD & MEFV & 0.455503 & Predicted \\
\hline CASP1 & PYCARD & 0.043769 & Predicted \\
\hline RELA & СЕВРВ & 0.024601 & Predicted \\
\hline NLRP3 & PYCARD & 0.25852 & Predicted \\
\hline CASP1 & CEBPB & 0.445416 & Predicted \\
\hline CASP1 & CEBPB & 0.707107 & Predicted \\
\hline PYCARD & MEFV & 0.00952 & Shared protein domains \\
\hline CASP1 & PYCARD & 0.013543 & Shared protein domains \\
\hline NLRP3 & MEFV & 0.009339 & Shared protein domains \\
\hline
\end{tabular}


TABLE 5: Continued.

\begin{tabular}{lccc}
\hline Gene 1 & Gene 2 & Weight & Network group \\
\hline NLRP3 & PYCARD & 0.018527 & Shared protein domains \\
NLRP14 & MEFV & 0.009512 & Shared protein domains \\
NLRP14 & PYCARD & 0.018871 & Shared protein domains \\
NLRP14 & NLRP3 & 0.036989 & Shared protein domains \\
ZNF528 & ZNF747 & 0.002699 & Shared protein domains \\
PYCARD & MEFV & 0.011528 & Shared protein domains \\
CASP1 & PYCARD & 0.031451 & Shared protein domains \\
NLRP3 & MEFV & 0.009427 & Shared protein domains \\
NLRP3 & PYCARD & 0.015448 & Shared protein domains \\
NLRP14 & MEFV & 0.009815 & Shared protein domains \\
NLRP14 & PYCARD & 0.016085 & Shared protein domains \\
NLRP14 & NLRP3 & 0.019774 & Shared protein domains \\
ZNF528 & ZNF747 & 0.002759 & Shared protein domains \\
\hline
\end{tabular}

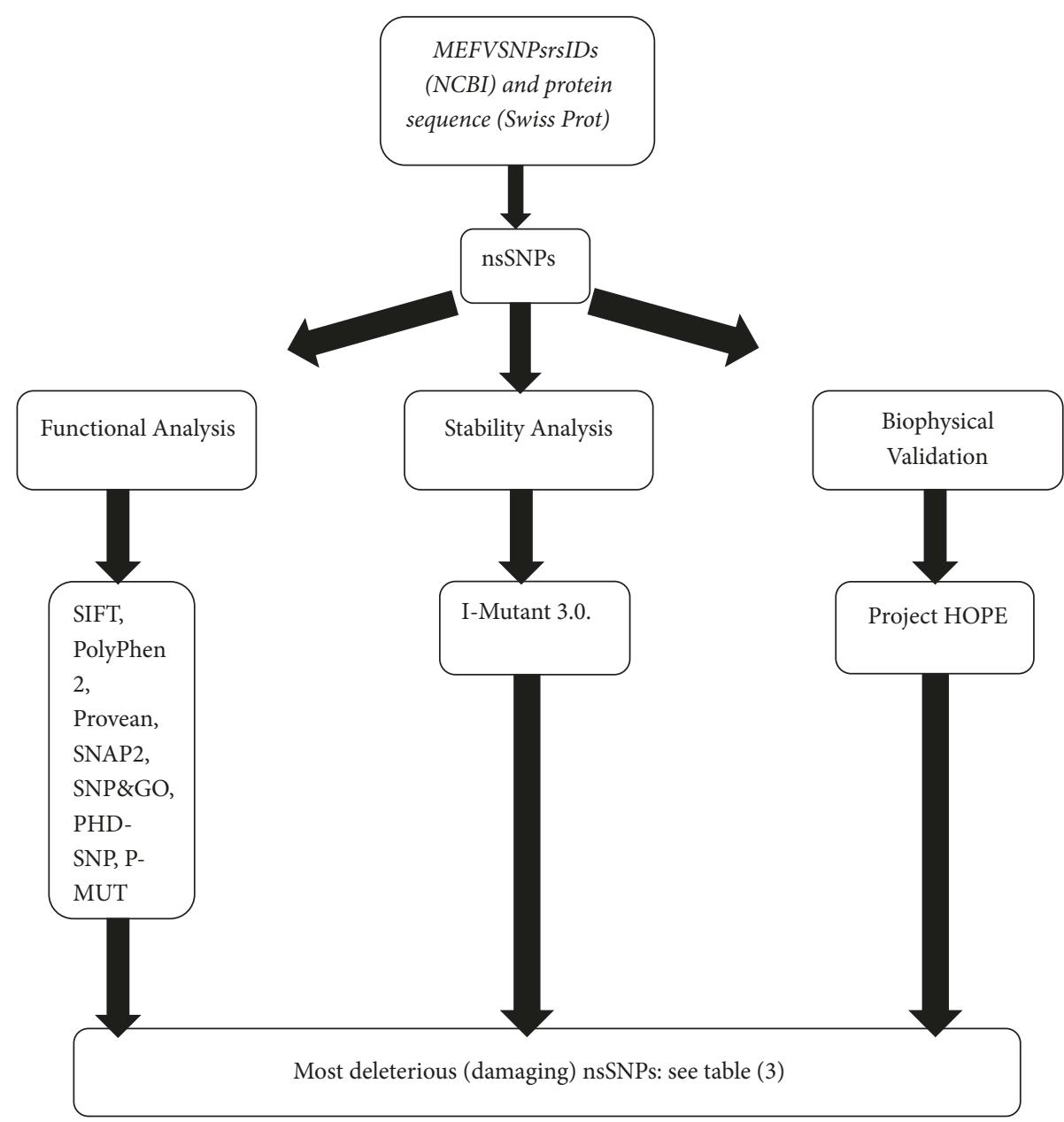

FIGURE 1: Diagrammatic representation of $M E F V$ gene in silico work flow.

intracellular receptor signaling pathway, nucleotide-binding domain, Leucine rich repeat containing receptor signaling pathway, positive regulation of cysteine-type endopeptidase activity, positive regulation of endopeptidase activity, positive regulation of peptidase activity, regulation of chemokine production, regulation of cysteine-type endopeptidase activity, regulation of endopeptidase activity, regulation of interleukin-1 beta production, regulation of interleukin1 production, and regulation of peptidase activity. The genes coexpressed with, sharing similar protein domain, or 


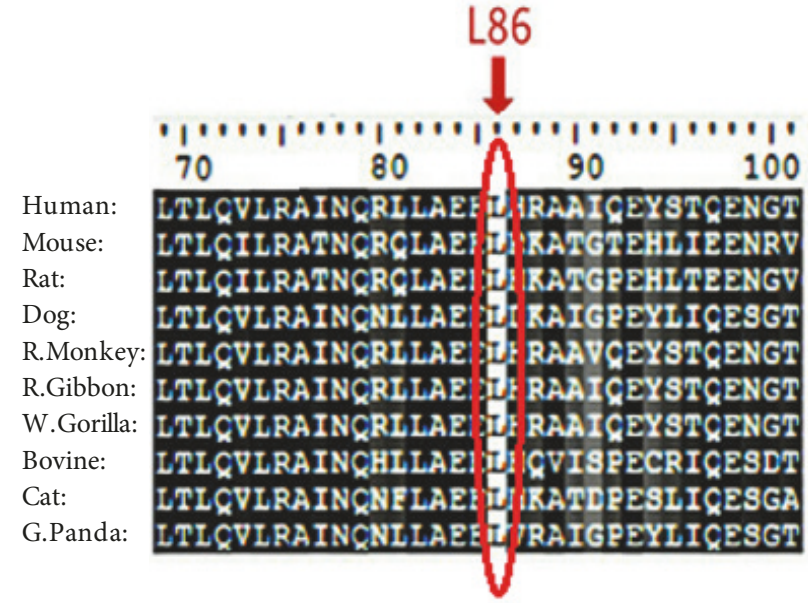

Figure 2: Alignments of 10 amino acid sequences of $M E F V$ demonstrating that the residues predicted to be mutated in our band (indicated by red arrow) are evolutionarily conserved across species. Sequences Alignment was done by BioEdit (v7.2.5).
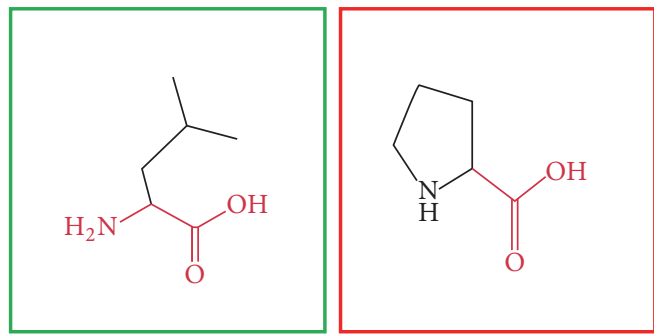

FIGURE 3: (L86P): change in the amino acid Leucine (green box) into Proline (red box) at position 86 .
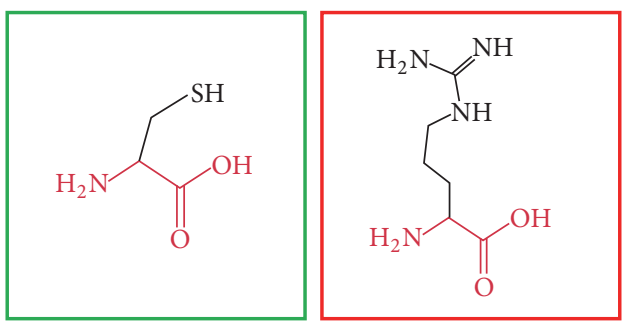

FIgURE 4: (C375R): change in the amino acid Cysteine (green box) into Arginine (red box) at position 375.
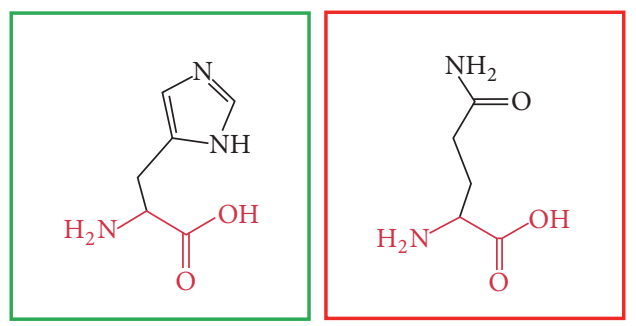

FIGURE 5: (H378Y): change in the amino acid Histidine (green box) into Tyrosine (red box) at position 378 .

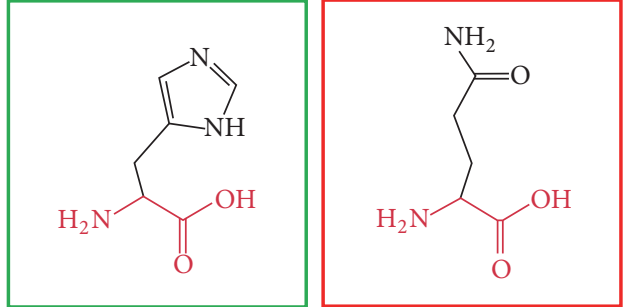

FIgURE 6: (H378Q): change in the amino acid Histidine (green box) into Glutamine (red box) at position 378 .

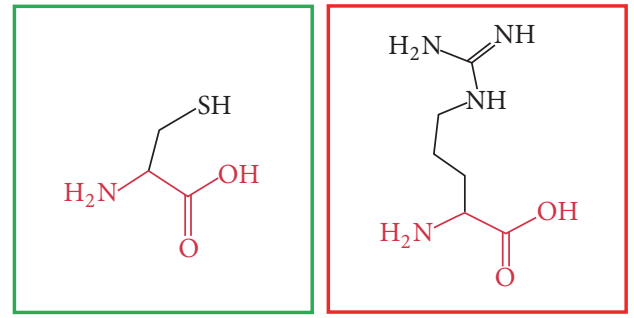

FIGURE 7: (C395R): change in the amino acid Cysteine (green box) into Arginine (red box) at position 395.
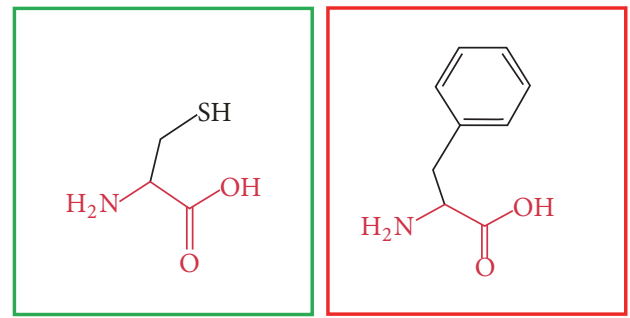

FIgURE 8: (C395F): change in the amino acid Cysteine (green box) into Phenylalanine (red box) at position 395.
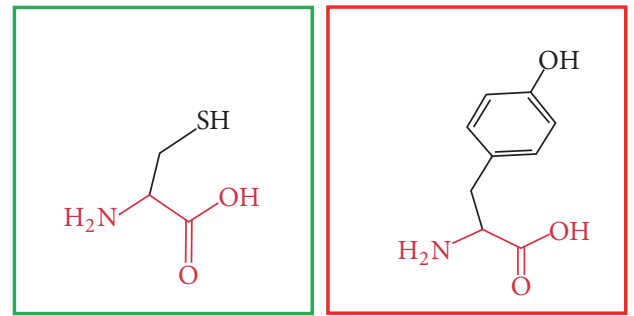

FIGURE 9: (C395Y): change in the amino acid Cysteine (green box) into Tyrosine (red box) at position 395.
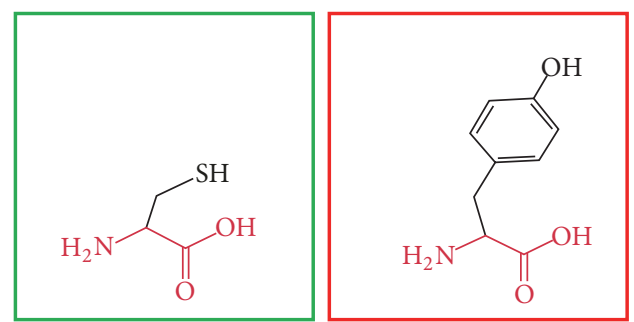

Figure 10: (C398Y): change in the amino acid Cysteine (green box) into Tyrosine (red box) at position 398. 

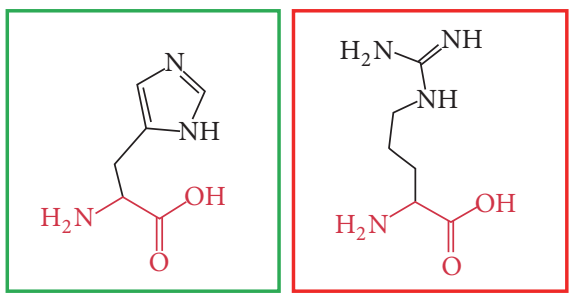

FIGURE 11: (H404R): change in the amino acid Histidine (green box) into Arginine (red box) at position 404.
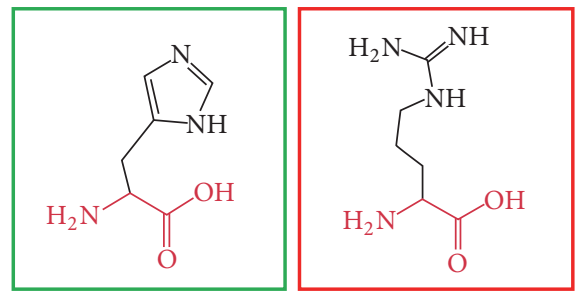

FIGURE 12: (H407R): change in the amino acid Histidine (green box) into Arginine (red box) at position 407.
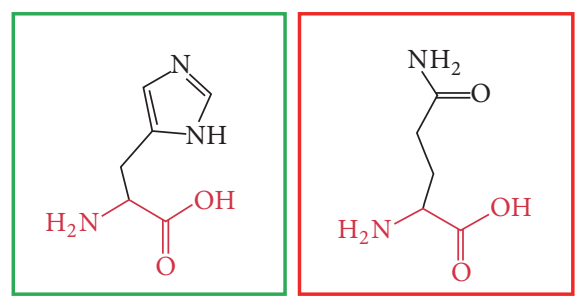

FIGURE 13: (H407Q): change in the amino acid Histidine (green box) into Glutamine (red box) at position 407.
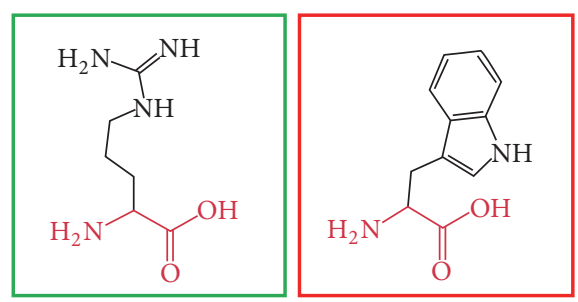

FIGURE 14: (R461W): change in the amino acid Arginine (green box) into Tryptophan (red box) at position 461.

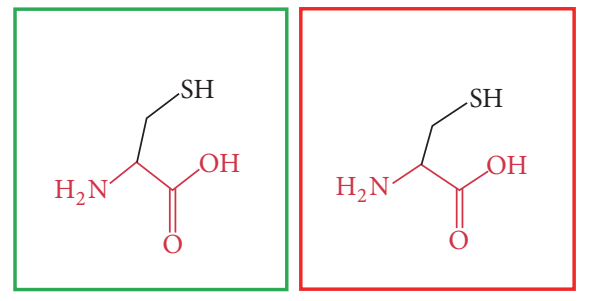

FIGURE 15: (F636C): change in the amino acid Phenylalanine (green box) into Cysteine (red box) at position 636.

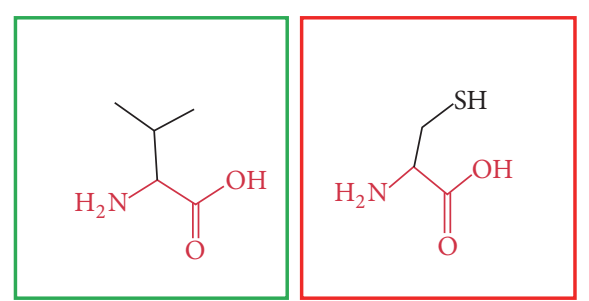

FIGURE 16: (V659F): change in the amino acid Valine (green box) into Phenylalanine (red box) at position 636.

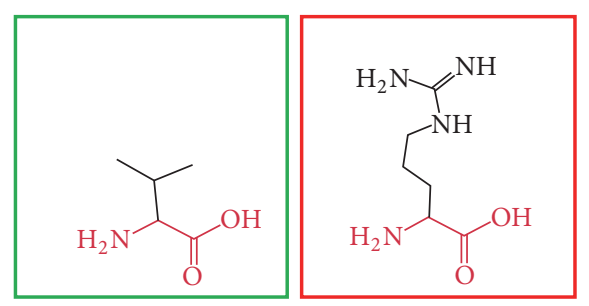

FIGURE 17: (G668R): change in the amino acid Glycine (green box) into Arginine (red box) at position 668 .
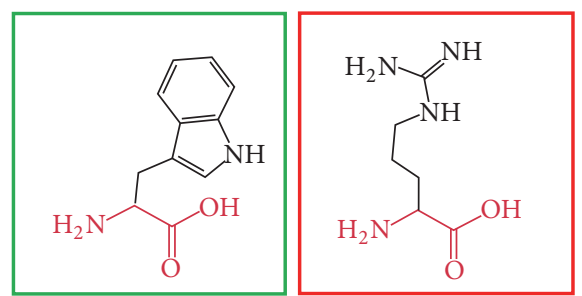

FIGURE 18: (W689R): change in the amino acid Tryptophan (green box) into Arginine (red box) at position 689.

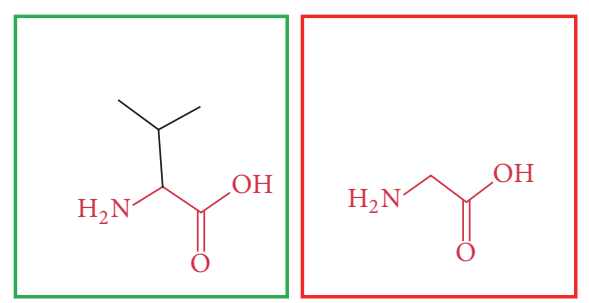

FIGURE 19: (V691G): change in the amino acid Valine (green box) into Glycine (red box) at position 691.
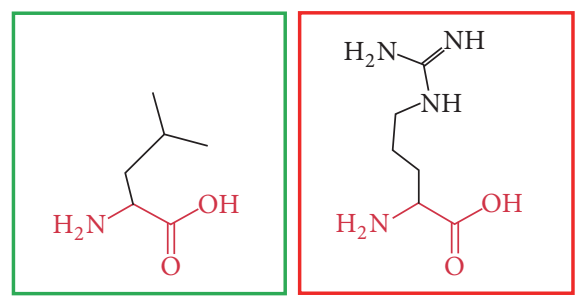

FIGURE 20: (L709R): change in the amino acid Leucine (green box) into Arginine (red box) at position 709. 


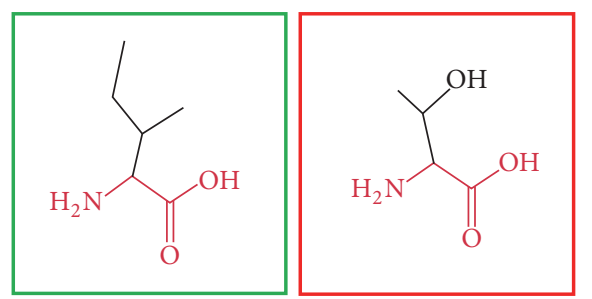

FIGURE 21: (I720T): change in the amino acid Isoleucine (green box) into Threonine (red box) at position 720 .

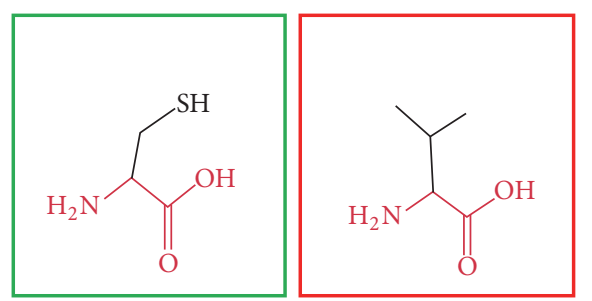

FIgURE 22: (F731V): change in the amino acid Phenylalanine (green box) into Valine (red box) at position 731 .

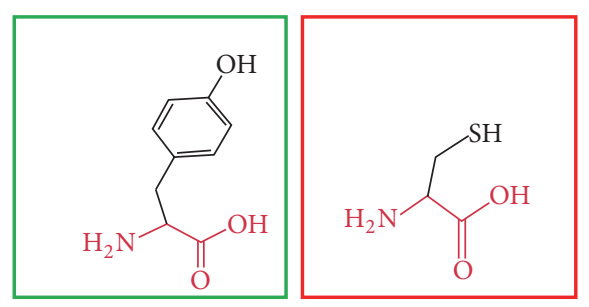

Figure 23: (Y741C): change in the amino acid Tyrosine (green box) into Cysteine (red box) at position 731.

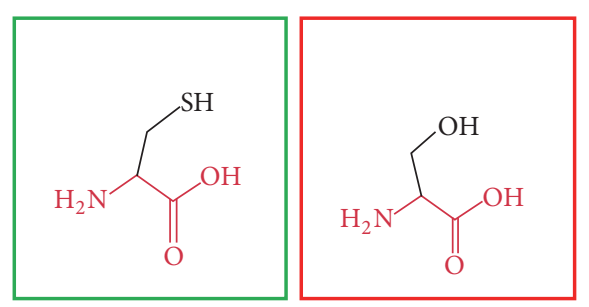

Figure 24: (F743S): change in the amino acid Phenylalanine (green box) into Serine (red box) at position 743 .

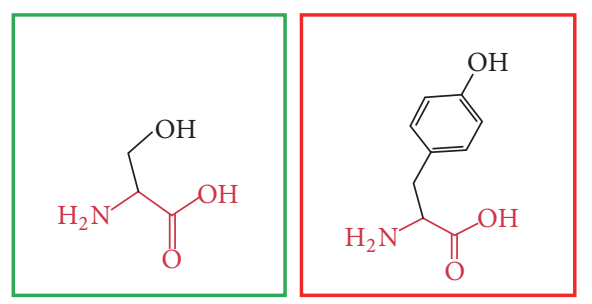

FIGURE 25: (S749Y): change in the amino acid Serine (green box) into Tyrosine (red box) at position 749 .

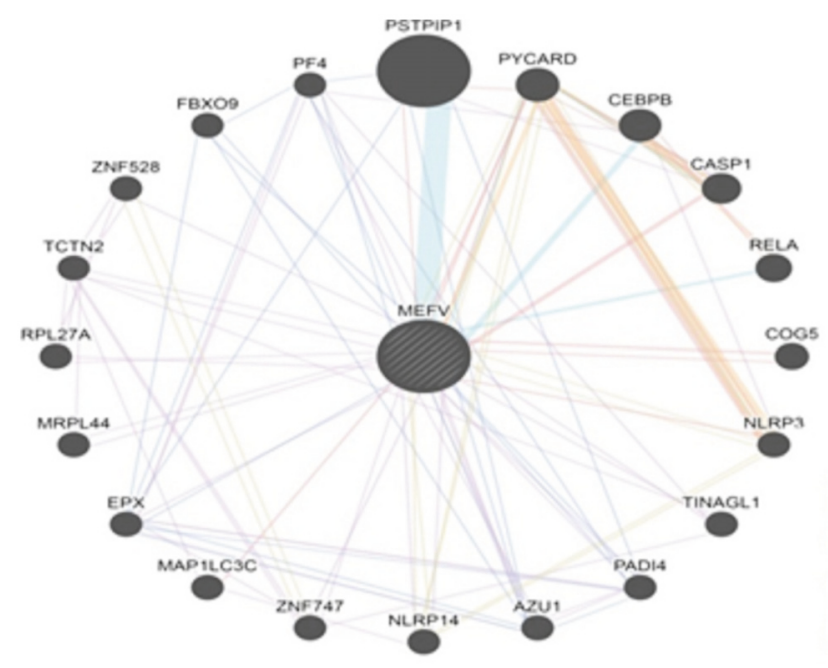

Networks

Physical Interactions

Co-expression

Predicted

Co-localization

Pathway

- Genetic Interactions

Shared protein domains

FIGURE 26: Interaction between $M E F V$ and its related genes.

participated to achieve similar function were shown in (see Figure 26) Tables 4 and 5.

In this study we also retrieved all these SNPs as untested (V659F, L709R, F743S, S749Y). We found it to be all damaging. Our study is the first in silico analysis of $M E F V$ gene which was based on functional analysis while all previous studies $[34,35]$ were based on frequency. This study revealed that 23 novel pathological mutations have a potential functional impact and may thus be used as diagnostic markers for Mediterranean basin populations.

\section{Conclusion}

In this work the influence of functional SNPs in the $M E F V$ gene was investigated through various computational methods, which determined that S749Y, F743S, Y741C, F731V, I720T, L709R, V691G, W689R, G668R, V659F, F636C, R461W, H407Q, H407R, H404R, C398Y, C395Y, C395F, C395R, H378Q, H378Y, C375R, and L86P are new SNPs having a potential functional impact and can thus be used as diagnostic markers. They constitute possible candidates for further genetic epidemiological studies with a special consideration of the large heterogeneity of MEFV SNPs among the different populations.

\section{Data Availability}

The data which support our findings in this study are available from the corresponding author upon reasonable request. 


\section{Conflicts of Interest}

The authors declare that there are no conflicts of interest regarding the publication of this paper.

\section{Authors' Contributions}

Mujahed I. Mustafa wrote Abstract, Methodology, and Result \& Discussion. Fatima A. Abdelrhman did Introduction. Conclusion was written by Soada A. Osman. Writing the original draft was carried out by Mujahed I. Mustafa.

\section{Acknowledgments}

The authors wish to acknowledge the enthusiastic cooperation of Africa City of Technology, Sudan.

\section{References}

[1] S. Georgin-Lavialle, V. Hentgen, K. Stankovic Stojanovic et al., "Familial Mediterranean fever," La Revue de Médecine Interne, vol. 39, no. 4, pp. 240-255, 2018.

[2] E. Ben-Chetrit and I. Touitou, "Familial mediterranean fever in the world," Arthritis \& Rheumatology, vol. 61, no. 10, pp. 14471453, 2009.

[3] E. Fragouli, E. Eliopoulos, E. Petraki et al., "Familial Mediterranean Fever in Crete: A genetic and structural biological approach in a population of 'intermediate risk,' Clinical Genetics, vol. 73, no. 2, pp. 152-159, 2008.

[4] M. G. Booty, J. C. Jae, S. L. Masters et al., "Familial Mediterranean fever with a single MEFV mutation: Where is the second hit?" Arthritis \& Rheumatology, vol. 60, no. 6, pp. 1851-1861, 2009.

[5] D. Marek-Yagel, Y. Berkun, S. Padeh et al., "Clinical disease among patients heterozygous for familial Mediterranean fever," Arthritis \& Rheumatology, vol. 60, no. 6, pp. 1862-1866, 2009.

[6] N. Ebadi, A. Shakoori, M. Razipour et al., "The spectrum of Familial Mediterranean Fever gene (MEFV) mutations and genotypes in Iran, and report of a novel missense variant (R204H)," European Journal of Medical Genetics, vol. 60, no. 12, pp. 701-705, 2017.

[7] N. Cekin, M. E. Akyurek, E. Pinarbasi, and F. Ozen, "MEFV mutations and their relation to major clinical symptoms of familial mediterranean fever," Gene, vol. 626, pp. 9-13, 2017.

[8] J. R. Al-Alami, M. K. Tayeh, D. A. Najib et al., "Familial mediterranean fever mutation frequencies and carrier rates among a mixed Arabic population," Saudi Medical Journal, vol. 24, no. 10, pp. 1055-1059, 2003.

[9] R. Gershoni-Baruch, M. Shinawi, K. Leah, K. Badarnah, and R. Brik, "Familial mediterranean fever: prevalence, penetrance and genetic drift," European Journal of Human Genetics, vol. 9, no. 8, pp. 634-637, 2001.

[10] N. Jalkh, E. Génin, E. Chouery et al., "Familial mediterranean fever in Lebanon: Founder effects for different MEFV mutations," Annals of Human Genetics, vol. 72, no. 1, pp. 41-47, 2008.

[11] N. Tomiyama, Y. Higashiuesato, T. Oda et al., "MEFV mutation analysis of familial Mediterranean fever in Japan," Clinical and Experimental Rheumatology, vol. 26, no. 1, pp. 13-17, 2008.

[12] R. Koshy, A. Sivadas, and V. Scaria, "Genetic epidemiology of familial Mediterranean fever through integrative analysis of whole genome and exome sequences from Middle East and North Africa," Clinical Genetics, vol. 93, no. 1, pp. 92-102, 2018.

[13] T. Yoldaş, Ş. Kayali, I. Ertuğrul, V. Doğan, U. A. Örün, and S. Karademir, "Massive pericardial effusion and tamponade can be a first sign of familial mediterranean fever," Pediatric Emergency Care, vol. 33, no. 9, pp. e48-e51, 2017.

[14] A. Slobodnick, B. Shah, S. Krasnokutsky, and M. H. Pillinger, "Update on colchicine, 2017," Rheumatology (Oxford), vol. 57, no. suppl_1, pp. i4-ill, 2018.

[15] A. Corsia, S. Georgin-Lavialle, V. Hentgen et al., "A survey of resistance to colchicine treatment for French patients with familial Mediterranean fever," Orphanet Journal of Rare Diseases, vol. 12, no. 1, p. 54, 2017.

[16] E. Verrecchia, L. L. Sicignano, M. La Regina et al., "Small intestinal bacterial overgrowth affects the responsiveness to colchicine in familial mediterranean fever," Mediators of Inflammation, vol. 2017, Article ID 7461426, 6 pages, 2017.

[17] S. Akar, P. Cetin, U. Kalyoncu et al., "Nationwide experience with off-label use of Interleukin-1 targeting treatment in familial mediterranean fever patients," Arthritis Care \& Research, vol. 70, no. 7, pp. 1090-1094, 2018.

[18] O. E. Gurkan, G. Yilmaz, A. U. Aksu, Z. Demirtas, G. Akyol, and B. Dalgic, "Colonic lymphoid nodular hyperplasia in childhood: Causes of familial mediterranean fever need extra attention," Journal of Pediatric Gastroenterology and Nutrition, vol. 57, no. 6, pp. 817-821, 2013.

[19] R. Heilig and P. Broz, "Function and mechanism of the pyrin inflammasome," European Journal of Immunology, vol. 48, no. 2, pp. 230-238, 2018.

[20] T. Kallinich, B. Orak, and H. Wittkowski, "Role of genetics in familial Mediterranean fever," Zeitschrift für Rheumatologie, vol. 76, no. 4, pp. 303-312, 2017.

[21] Y. Jamilloux, L. Lefeuvre, F. Magnotti et al., "Familial Mediterranean fever mutations are hypermorphic mutations that specifically decrease the activation threshold of the Pyrin inflammasome," Rheumatology, vol. 57, no. 1, pp. 100-111, 2018.

[22] S. Özen, E. D. Batu, and S. Demir, "Familial mediterranean fever: recent developments in pathogenesis and new recommendations for management," Frontiers in Immunology, vol. 8, p. 253, 2017.

[23] A. Bajard, S. Chabaud, C. Cornu et al., "An in silico approach helped to identify the best experimental design, population, and outcome for future randomized clinical trials," Journal of Clinical Epidemiology, vol. 69, pp. 125-136, 2016.

[24] D. A. Benson, I. Karsch-Mizrachi, K. Clark, D. J. Lipman, J. Ostell, and E. W. Sayers, "GenBank," Nucleic Acids Research, vol. 40, no. D1, pp. D48-D53, 2012.

[25] P. Artimo, M. Jonnalagedda, K. Arnold, D. Baratin, G. Csardi, E. De Castro et al., "ExPASy: SIB bioinformatics resource portal," Nucleic Acids Research, vol. 40, no. W1, pp. W597-W603, 2012.

[26] N.-L. Sim, P. Kumar, J. Hu, S. Henikoff, G. Schneider, and P. C. Ng, "SIFT web server: predicting effects of amino acid substitutions on proteins," Nucleic Acids Research, vol. 40, no. W1, pp. W452-W457, 2012.

[27] I. A. Adzhubei, S. Schmidt, L. Peshkin et al., "A method and server for predicting damaging missense mutations," Nature Methods, vol. 7, no. 4, pp. 248-249, 2010.

[28] Y. Choi, G. E. Sims, S. Murphy, J. R. Miller, and A. P. Chan, "Predicting the functional effect of amino acid substitutions and indels," PLoS ONE, vol. 7, no. 10, Article ID e46688, 2012. 
[29] M. Hecht, Y. Bromberg, and B. Rost, "Better prediction of functional effects for sequence variants," BMC Genomics, vol. 16, no. Suppl 8, p. S1, 2015.

[30] R. Calabrese, E. Capriotti, P. Fariselli, P. L. Martelli, and R. Casadio, "Functional annotations improve the predictive score of human disease-related mutations in proteins," Human Mutation, vol. 30, no. 8, pp. 1237-1244, 2009.

[31] V. López-Ferrando, A. Gazzo, X. De La Cruz, M. Orozco, and J. L. Gelpí, "PMut: A web-based tool for the annotation of pathological variants on proteins, 2017 update," Nucleic Acids Research, vol. 45, no. W1, pp. W222-W228, 2017.

[32] E. Capriotti, P. Fariselli, and R. Casadio, "I-Mutant2.0: predicting stability changes upon mutation from the protein sequence or structure," Nucleic Acids Research, vol. 33, no. suppl_2, pp. W306-W310, 2005.

[33] D. W. Farley, S. L. Donaldson, O. Comes et al., "The GeneMANIA prediction server: biological network integration for gene prioritization and predicting gene function," Nucleic Acids Research, vol. 38, no. 2, pp. W214-W220, 2010.

[34] E. Gumus, "The frequency of MEFV gene mutations and genotypes in Sanliurfa province, South-Eastern region of Turkey, after the Syrian Civil War by using next generation sequencing and report of a Novel Exon 4 Mutation (I423T)," Journal of Clinical Medicine, vol. 7, no. 5, p. 105, 2018.

[35] M. Medlej-Hashim, J.-L. Serre, S. Corbani et al., "Familial Mediterranean fever (FMF) in Lebanon and Jordan: a population genetics study and report of three novel mutations," European Journal of Medical Genetics, vol. 48, no. 4, pp. 412420, 2005. 


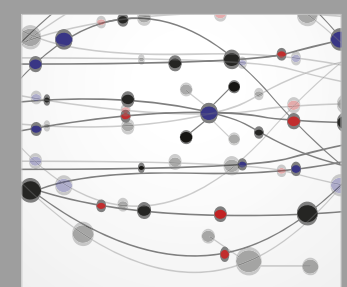

The Scientific World Journal
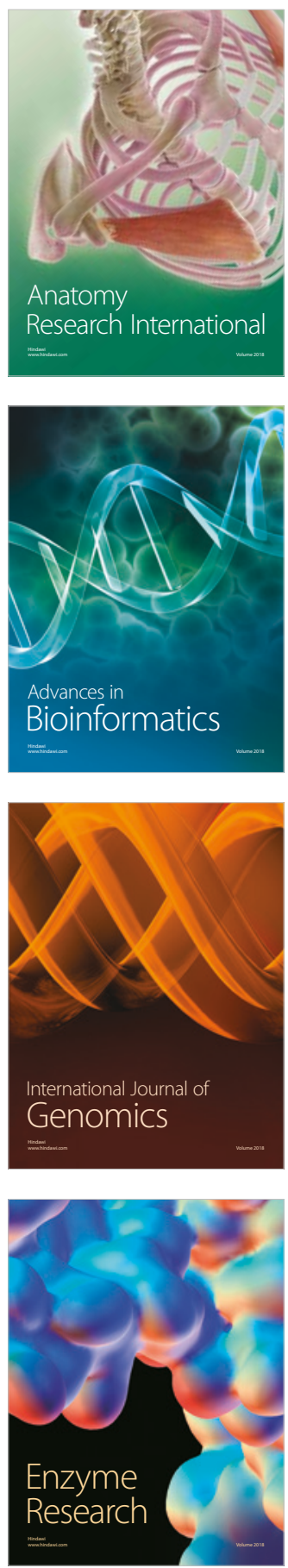
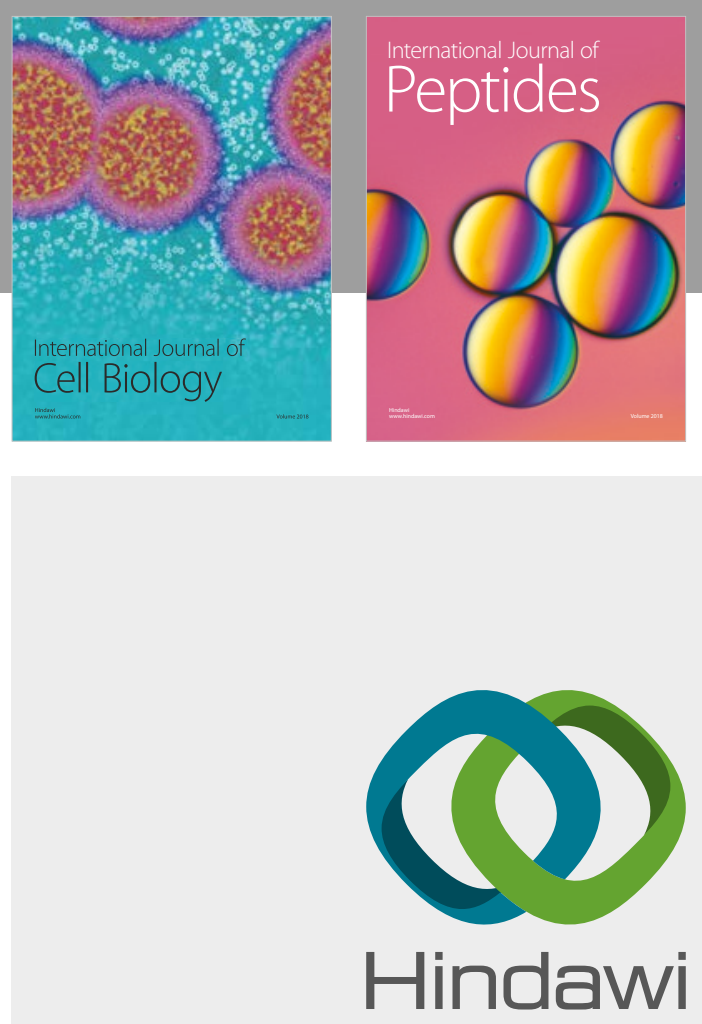

Submit your manuscripts at

www.hindawi.com
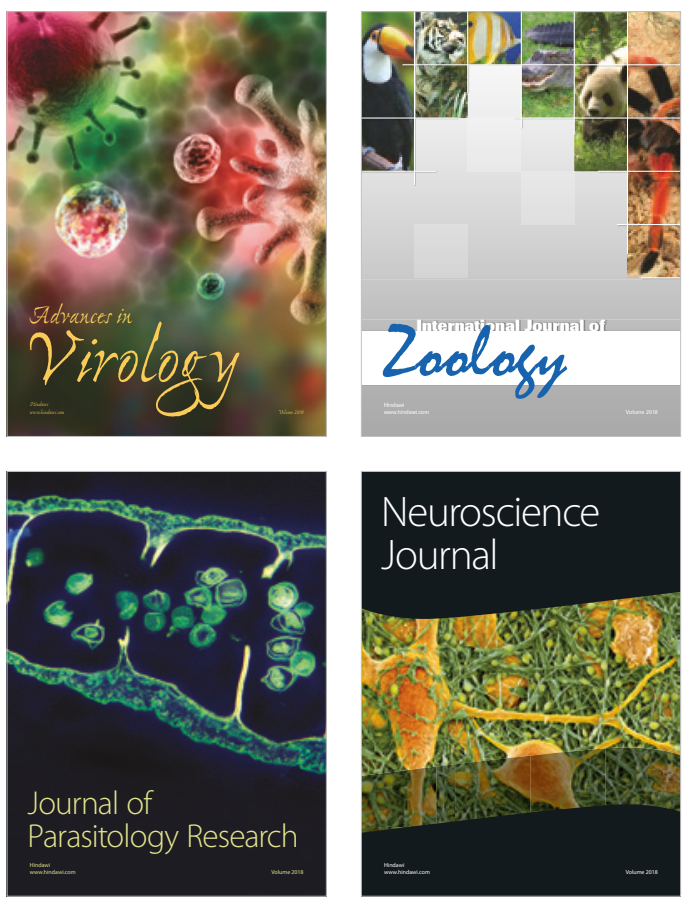
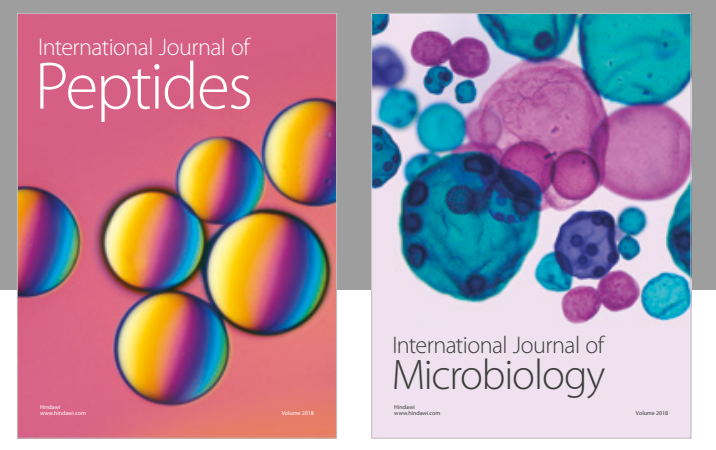

nternational Journal of Microbiology
Journal of
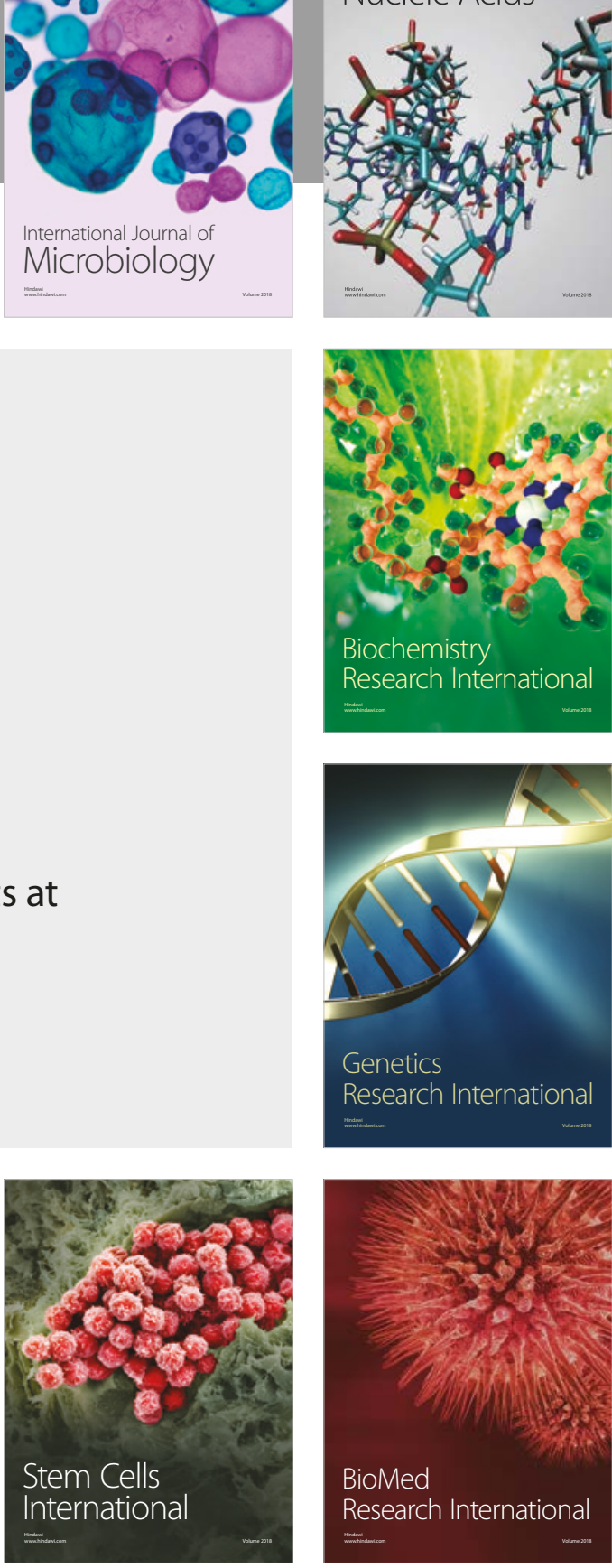
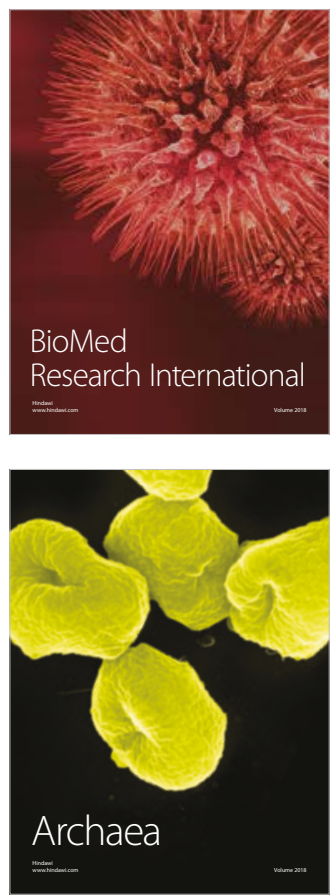